\title{
Entropies of the partitions of the unit interval generated by the Farey tree
}

\author{
by \\ Nikolai Moshchevitin (Moscow) and \\ Anatoly ZhiglJaVsky (Cardiff)
}

1. Introduction. The Farey tree $\mathcal{F}$ is an infinite binary tree whose nodes are labelled by rationals in $[0,1]$; it can be defined inductively as follows.

Consider the two-point set $\mathcal{F}_{1}=\{0,1\}$ with 0 and 1 written as $\frac{0}{1}$ and $\frac{1}{1}$ respectively. Let $n \geq 1$ and $0=x_{0, n}<x_{1, n}<\cdots<x_{N(n), n}=1$ be the fractions in $\mathcal{F}_{n}$ arranged in order of increase and written in lowest terms; here $N(n)=2^{n-1}$. Then

$$
\mathcal{F}_{n+1}=\mathcal{F}_{n} \cup \mathcal{Q}_{n+1} \quad \text { with } \quad \mathcal{Q}_{n+1}=\left\{x_{i-1, n} \oplus x_{i, n}, i=1, \ldots, 2^{n-1}\right\},
$$

where

$$
\frac{p}{q} \oplus \frac{p^{\prime}}{q^{\prime}}=\frac{p+p^{\prime}}{q+q^{\prime}}
$$

is the mediant of the fractions $\frac{p}{q}$ and $\frac{p^{\prime}}{q^{\prime}}$. For example,

$$
\mathcal{F}_{2}=\left\{0, \frac{1}{2}, 1\right\}, \quad \mathcal{F}_{3}=\left\{0, \frac{1}{3}, \frac{1}{2}, \frac{2}{3}, 1\right\}, \quad \mathcal{F}_{4}=\left\{0, \frac{1}{4}, \frac{1}{3}, \frac{2}{5}, \frac{1}{2}, \frac{3}{5}, \frac{2}{3}, \frac{3}{4}, 1\right\} .
$$

$\mathcal{F}_{n}$ is sometimes called the Brocot sequence of order $n$. The elements of $\mathcal{Q}_{n}$ are known as Farey fractions of level $n$; for $n \geq 2$ they are the nodes in the Farey tree at level $n-1$. The first (root) node of the tree is $\frac{1}{2}$.

It is well known (see e.g. Schroeder (1991), p. 337) and straightforward to check that for the Farey fractions of level $n$ the sum of partial quotients in their continued fraction representation is exactly $n$; that is,

$$
\begin{array}{r}
\mathcal{Q}_{n}=\left\{\frac{p}{q}=1 /\left(a_{1}+1 /\left(a_{2}+\cdots+1 / a_{t}\right) \ldots\right)\right. \\
\text { with } \left.a_{t} \geq 2 \text { and } a_{1}+\cdots+a_{t}=n\right\} .
\end{array}
$$

2000 Mathematics Subject Classification: 11J70, 11K60, $11 \mathrm{D} 68$.

Key words and phrases: Farey tree, continued fractions, transfer operator, Farey map, partition of the interval.

The first author was partly supported by Russian Science Foundation grants RFFI 02-01-00912, NSh-136.2003.1 and MD-3321.2004.1 as well as INTAS grant 03-51-5070. 
Fractions $x_{i, n} \in \mathcal{F}_{n}$ considered as points in $[0,1]$ make a partition of this interval into $2^{n-1}$ subintervals of different length varying from $1 /\left(F_{n} F_{n+1}\right)$ to $1 / n$, where $F_{k}$ is the $k$ th Fibonacci number. This partition is obviously non-uniform; in this paper we study the asymptotic behaviour of a natural characteristic that measures this non-uniformity and is defined as follows.

Let $0=x_{0, n}<x_{1, n}<\cdots<x_{N(n), n}=1$ be some points in $[0,1]$ and $p_{i, n}=x_{i, n}-x_{i-1, n}(i=1, \ldots, N(n))$ be the lengths of the subintervals $\left[x_{i-1, n}, x_{i, n}\right)$. For a fixed $\beta$ we set

$$
\sigma_{\beta}^{(n)}=\sigma\left(x_{0, n}, \ldots, x_{N(n), n}\right)=\sum_{i=1}^{N(n)} p_{i, n}^{\beta} .
$$

$\sigma_{\beta}^{(n)}$ is one of the most widely used characteristics of the uniformity of the partition of $[0,1]$ generated be the points $x_{i, n}$ (see e.g. Drobot (1981)). The value $\frac{1}{1-\beta} \log \sigma_{\beta}^{(n)}$ is the Rényi entropy of order $\beta \neq 1$ of this partition (as $\beta \rightarrow 1$ the Rényi entropies tend to the Shannon entropy of the partition). If the partition is defined by a dynamical system, then the properly normalised sequence of $\sigma_{\beta}^{(n)}$ converges to the maximum eigenvalue of the transfer operator (3) (see e.g. Vallée (2001)). In an important special case $\beta=2$ the quantity (1) can be interpreted as the average length of the interval $\left[x_{i-1, n}, x_{i, n}\right)$ which a random uniformly distributed point in $[0,1]$ falls in (see Section 4.2 in Pronzato et al. (1999) for details).

In a number of papers including Hall (1970) and Kanemitsu et al. (1982), the limiting behaviour of $\sigma_{\beta}^{(n)}$ is studied in the case when $\left\{x_{i, n}\right\}$ is the Farey sequence of order $n$, that is, the set of all fractions $p / q$ in $[0,1]$ with $\operatorname{gcd}(p, q)=1$ and $q \leq n$. In the present paper we study the limiting behaviour of $\sigma_{\beta}^{(n)}$ when $x_{i, n}$ are the elements of $\mathcal{F}_{n}$; in this case we will write $\sigma_{\beta}^{(n)}=\sigma_{\beta}\left(\mathcal{F}_{n}\right)$.

The expression for $\sigma_{\beta}\left(\mathcal{F}_{n}\right)$ can be simplified using the fact that if $\frac{p}{q}$ and $\frac{p^{\prime}}{q^{\prime}}$ are two neighbours in $\mathcal{F}_{n}$ such that $\frac{p}{q}<\frac{p^{\prime}}{q^{\prime}}$, then the length of the interval $\left[\frac{p}{q}, \frac{p^{\prime}}{q^{\prime}}\right)$ is $p^{\prime} / q^{\prime}-p / q=1 /\left(q q^{\prime}\right)$. This yields

$$
\sigma_{\beta}\left(\mathcal{F}_{n}\right)=\sum_{\left(q, q^{\prime}\right)} \frac{1}{\left(q q^{\prime}\right)^{\beta}},
$$

where the sum is taken over the set of pairs of denominators of all the neighbours in $\mathcal{F}_{n}$.

The following theorem is the main result of this paper. 
TheOrem 1. For any $\beta>1$ we have

$$
\sigma_{\beta}\left(\mathcal{F}_{n}\right)=\frac{2}{n^{\beta}} \cdot \frac{\zeta(2 \beta-1)}{\zeta(2 \beta)}+O\left(\frac{\log n}{n^{(\beta+1)(2 \beta-1) /(2 \beta)}}\right) \quad \text { as } n \rightarrow \infty .
$$

The proof of the theorem is given in Section 3 .

Note that for small $\beta>1$ the rate of convergence in (2) is slow. However, the fact that for all $\beta>1$ the main term for $\sigma_{\beta}\left(\mathcal{F}_{n}\right)$ is indeed $\frac{2}{n^{\beta}} \frac{\zeta(2 \beta-1)}{\zeta(2 \beta)}$ agrees with numerical experiments that the authors have carried out. As an example, Table 1 illustrates the rate of convergence in (2) for $\beta=2$, where the main term in the asymptotic formula (2) is $\frac{2 \zeta(3)}{\zeta(4)} n^{-2} \cong 2.22125 n^{-2}$.

Table 1. Numerically computed values of $\widetilde{\sigma}_{n}=n(n-1) \sigma_{2}\left(\mathcal{F}_{n}\right)$ for $n=2, \ldots, 41$

\begin{tabular}{cc|cc|cc|cc}
\hline$n$ & $\widetilde{\sigma}_{n}$ & $n$ & $\widetilde{\sigma}_{n}$ & $n$ & $\widetilde{\sigma}_{n}$ & $n$ & $\widetilde{\sigma}_{n}$ \\
\hline 2 & 1 & 12 & 2.225125138 & 22 & 2.198914837 & 32 & 2.199080361 \\
3 & 1.666666667 & 13 & 2.218892938 & 23 & 2.198552286 & 33 & 2.199321142 \\
4 & 2.013333333 & 14 & 2.213886731 & 24 & 2.198328443 & 34 & 2.199573834 \\
5 & 2.172902494 & 15 & 2.209932920 & 25 & 2.198214857 & 35 & 2.199835181 \\
6 & 2.237347594 & 16 & 2.206846310 & 26 & 2.198188931 & 36 & 2.200102532 \\
7 & 2.257088762 & 17 & 2.204459272 & 27 & 2.198232662 & 37 & 2.200373722 \\
8 & 2.257418374 & 18 & 2.202630388 & 28 & 2.198331652 & 38 & 2.200646994 \\
9 & 2.250363055 & 19 & 2.201244461 & 29 & 2.198474352 & 39 & 2.200920916 \\
10 & 2.241355171 & 20 & 2.200209351 & 30 & 2.198651468 & 40 & 2.201194325 \\
11 & 2.232670354 & 21 & 2.199452029 & 31 & 2.198855495 & 41 & 2.201466280 \\
\hline
\end{tabular}

The order of decrease for $\sigma_{\beta}\left(\mathcal{F}_{n}\right)$ as $n \rightarrow \infty$ is different from $n^{-\beta}$ for $\beta \leq 1$. Thus, $\sigma_{0}\left(\mathcal{F}_{n}\right)=2^{n-1}$ (this is the number of intervals in the $n$th level partition) and $\sigma_{1}\left(\mathcal{F}_{n}\right)=1$ (see Lemma 2$)$.

\section{Reformulation of the problem in terms of dynamical systems and continued fractions}

2.1. Reformulation in terms of dynamical systems. The Farey map $T$ : $[0,1] \rightarrow[0,1]$ is defined by

$$
T(x)= \begin{cases}x /(1-x) & \text { if } 0 \leq x<1 / 2, \\ (1-x) / x & \text { if } 1 / 2 \leq x \leq 1\end{cases}
$$


The map is shown in Figure 1.

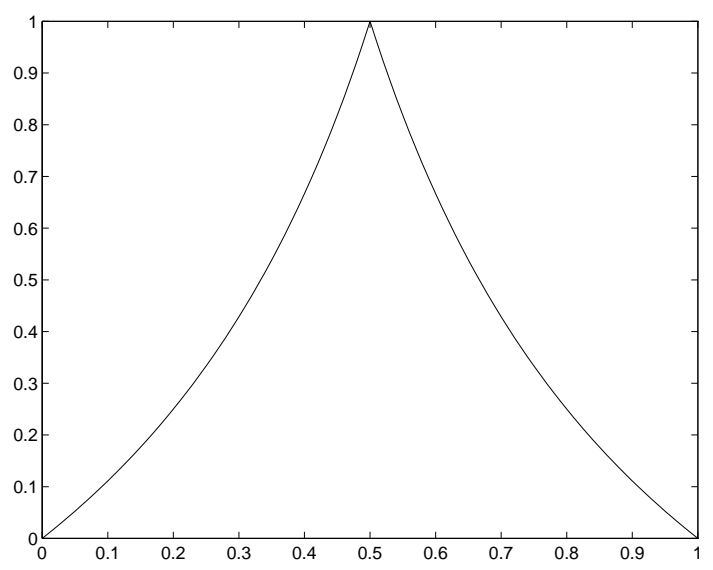

Fig. 1. The Farey map

There is a simple relation between the Farey fractions of level $n$ and the Farey map:

$$
\mathcal{Q}_{n}=T^{-n+1}(1)=\left\{x \in[0,1]: T^{n-1}(x)=1\right\} \quad \forall n \geq 2,
$$

implying $\mathcal{F}_{n}=T^{-n}(0)$ for all $n \geq 1$.

The Farey map belongs to a class of so-called almost expanding maps. It has absolutely continuous invariant density $p(x)=1 / x(0<x<1)$ and it is ergodic with respect to this density; the density $p(x)$ has infinite mass implying that the metric entropy of $T(\cdot)$ is zero (for details see e.g. Lagarias (1992)). Moreover, the topological pressure $P_{\beta}$ of the Farey map is zero for $\beta \geq 1$ (see Prellberg and Slawny (1992)). The pressure can be defined as $P_{\beta}=\log \lambda_{\beta}$, where $\lambda_{\beta}$ is the maximal eigenvalue of the transfer operator $\mathcal{L}_{\beta}: C[0,1] \rightarrow C[0,1]$ defined for $f \in C[0,1]$ by

$$
\mathcal{L}_{\beta} f(x)=\sum_{y: T(y)=x} \frac{f(y)}{\left|T^{\prime}(y)\right|^{\beta}} .
$$

For the Farey map the pressure is

$$
P_{\beta}=\lim _{n \rightarrow \infty} \frac{1}{n} \log \sum_{\left(q, q^{\prime}\right)} \frac{1}{\left(q q^{\prime}\right)^{\beta}},
$$

where for fixed $n$ the sum is taken over the set of pairs of denominators of all the neighbours in $\mathcal{F}_{n}$.

Prellberg and Slawny (1992) studied the behaviour of the pressure $P_{\beta}$ for a class of almost expanding maps (including the Farey map) as $\beta \uparrow 1$; we consider a version of the pressure for $\beta>1$. Theorem 1 shows that to obtain 
non-trivial limits, the normalisation of the sum in (4) with $\beta>1$ must be different from $\frac{1}{n} \log$; this normalization is $n^{\beta}$. A similar phenomenon seems to hold for some other almost expanding maps; this phenomenon is related to the non-exponential divergence of the trajectories $x_{n+1}=T\left(x_{n}\right)$ for those maps.

2.2. Reformulation in terms of continued fractions. Let $\mathcal{A}$ be the set of all integer vectors $a=\left(a_{1}, \ldots, a_{t}\right)$ with $t \geq 1, a_{j} \geq 1(j=1, \ldots, t-1)$ and $a_{t}>1$. Let also

$$
\mathcal{A}_{n}=\left\{a=\left(a_{1}, \ldots, a_{t}\right) \in \mathcal{A}: a_{1}+\cdots+a_{t}=n\right\} .
$$

With each $a=\left(a_{1}, \ldots, a_{t}\right) \in \mathcal{A}$ we associate the continued fraction $1 /\left(a_{1}+1 /\left(a_{2}+\cdots+1 / a_{t}\right) \ldots\right)$ and the corresponding continuant (the denominator of the fraction), which we write as $\left[a_{1}, \ldots, a_{t}\right]$; an empty continuant is equal to 1 .

By construction, for all $n>1$, each fraction in $\mathcal{F}_{n} \backslash\left(\mathcal{F}_{1} \cup Q_{n}\right)$ has two neighbours which belong to the set $\mathcal{Q}_{n}$. Also, every fraction $p / q \in \mathcal{Q}_{n}$ has two neighbours, say $p_{-} / q_{-}$and $p_{+} / q_{+}$, in $\mathcal{F}_{n} \backslash \mathcal{Q}_{n}$. Explicit formulae for the continuants of these neighbours are given below.

Lemma 1. For each $a \in \mathcal{A}_{n}$, the fraction $p / q \in \mathcal{Q}_{n}$ with continuant $q=q(a)=\left[a_{1}, \ldots, a_{t}\right]$ has two neighbours in $\mathcal{F}_{n}$ with continuants

$$
q_{-}=q_{-}(a)=\left[a_{1}, \ldots, a_{t-1}\right], \quad q_{+}=q_{+}(a)=\left[a_{1}, \ldots, a_{t}-1\right] .
$$

Similarly, any fraction $p / q \in \mathcal{F}_{n-1} \backslash \mathcal{F}_{1}$ with continuant $q=q(a)=$ $\left[a_{1}, \ldots, a_{t}\right]$ has two neighbours in $\mathcal{F}_{n}$ with continuants

$$
\left[a_{1}, \ldots, a_{t}, n-\left(a_{1}+\cdots+a_{t}\right)\right], \quad\left[a_{1}, \ldots, a_{t}-1,1, n-\left(a_{1}+\cdots+a_{t}\right)\right] .
$$

The proof is a simple induction with respect to $n$.

Note that the two neighbours of the fraction $p / q$ with continuants $q_{-}$ and $q_{+}$are not simply left and right: the larger denominator $q_{+}$can be on either side of $q$.

The first part of Lemma 1 implies that we can rewrite $\sigma_{\beta}\left(\mathcal{F}_{n}\right)$, the characteristic of interest, as

$$
\sigma_{\beta}\left(\mathcal{F}_{n}\right)=\sum_{a \in \mathcal{A}_{n}}\left(\frac{1}{\left(q q_{-}\right)^{\beta}}+\frac{1}{\left(q q_{+}\right)^{\beta}}\right),
$$

where $q=q(a)=\left[a_{1}, \ldots, a_{t}\right]$ and $q_{-}=q_{-}(a), q_{+}=q_{+}(a)$ are defined in (5).

For any $\beta>1$ we can easily establish a lower bound for $\sigma_{\beta}\left(\mathcal{F}_{n}\right)$ in the following way: 


$$
\begin{aligned}
\sigma_{\beta}\left(\mathcal{F}_{n}\right) & =\sum_{a \in \mathcal{A}_{n}}\left(\frac{1}{\left(q q_{-}\right)^{\beta}}+\frac{1}{\left(q q_{+}\right)^{\beta}}\right) \geq \sum_{a \in \mathcal{A}_{n}} \frac{1}{\left(q q_{-}\right)^{\beta}} \geq \frac{1}{n^{\beta}} \sum_{a \in \mathcal{A}_{n}} \frac{1}{\left(q_{-}\right)^{2 \beta}} \\
& \geq \frac{1}{n^{\beta}} \sum_{a \in \mathcal{A}_{n}: q_{-}<n} \frac{1}{\left(q_{-}\right)^{2 \beta}}=\frac{2}{n^{\beta}} \sum_{q_{-}=1}^{n-1} \frac{\varphi\left(q_{-}\right)}{\left(q_{-}\right)^{2 \beta}} \\
& =\frac{2}{n^{\beta}} \sum_{l=1}^{\infty} \frac{\varphi(l)}{l^{2 \beta}}+O\left(\frac{1}{n^{2 \beta-1}}\right) \quad \text { as } n \rightarrow \infty .
\end{aligned}
$$

In deriving the lower bound we have neglected the terms with large denominators $q_{-}(a)$, used the inequality $q \leq n q_{-}$(see (10)) and the fact that in the partition generated by $\mathcal{F}_{n}$ there are exactly $2 \varphi(l)$ intervals with $q_{-}(a)=l<n$ as one of their end-points.

Theorem 1 states that the just derived lower bound

$$
\liminf _{n \rightarrow \infty} n^{\beta} \sigma_{\beta}\left(\mathcal{F}_{n}\right) \geq 2 \sum_{l=1}^{\infty} \frac{\varphi(l)}{l^{2 \beta}}=\frac{2 \zeta(2 \beta-1)}{\zeta(2 \beta)}
$$

is in reality the exact limit of $n^{\beta} \sigma_{\beta}\left(\mathcal{F}_{n}\right)$ as $n \rightarrow \infty$. Thus, the main contribution to the sum $\sigma_{\beta}\left(\mathcal{F}_{n}\right)$ is made by a few terms with small denominators $q_{-}$("major arcs").

\section{Proof of Theorem 1}

3.1. More notation and the main steps in the proof. Throughout this section we assume that $\beta>1$ is a fixed number. Set

$$
r=r(\beta)=\frac{3 \beta-2}{2(\beta-1)}, \quad w=w(n, \beta)=\min \left\{n / 2, n^{(\beta+1) /(2 \beta)} \log n\right\} .
$$

The value of $r$ is chosen to satisfy $(\beta-1)(2 r-1)=2 \beta-1$ (see Lemma 3$)$; $w=w(n, \beta)$ will minimize the error term. Note that since $\beta>1$ we have $w=o(n)$ as $n \rightarrow \infty$.

For a subset $\mathcal{A}_{n, i}^{(j)}$ of $\mathcal{A}$, define

$$
\Sigma_{n, i}^{(j)}=\sum_{a \in \mathcal{A}_{n, i}^{(j)}}\left(\frac{1}{\left(q q_{-}\right)^{\beta}}+\frac{1}{\left(q q_{+}\right)^{\beta}}\right),
$$

where $a=\left(a_{1}, \ldots, a_{t}\right), q=q(a)=\left[a_{1}, \ldots, a_{t}\right] ; q_{-}=q_{-}(a)$ and $q_{+}=q_{+}(a)$ are defined in (5).

The proof of Theorem 1 is based on a few lemmas and uses the splitting of the sum $\sigma_{\beta}\left(\mathcal{F}_{n}\right)$, which is the sum over $\mathcal{A}_{n}$, into the sums $\Sigma_{n, i}^{(j)}$ over smaller 
subsets of indices $a$. The first split of $\mathcal{A}_{n}$ is into the following two subsets:

$$
\begin{aligned}
& \mathcal{A}_{n, 1}^{(1)}=\left\{a=\left(a_{1}, \ldots, a_{t}\right) \in \mathcal{A}_{n}:\left[a_{1}, \ldots, a_{t}\right]<n^{r}\right\}, \\
& \mathcal{A}_{n, 2}^{(1)}=\mathcal{A}_{n} \backslash \mathcal{A}_{n, 1}^{(1)}=\left\{a=\left(a_{1}, \ldots, a_{t}\right) \in \mathcal{A}_{n}:\left[a_{1}, \ldots, a_{t}\right] \geq n^{r}\right\} .
\end{aligned}
$$

We then split $\mathcal{A}_{n, 1}^{(1)}$ into two subsets as follows:

$$
\begin{aligned}
& \mathcal{A}_{n, 1}^{(2)}=\left\{a \in \mathcal{A}_{n, 1}^{(1)}: \max _{1 \leq j \leq t} a_{j}>n-w\right\}, \\
& \mathcal{A}_{n, 2}^{(2)}=\mathcal{A}_{n, 1}^{(1)} \backslash \mathcal{A}_{n, 1}^{(2)}=\left\{a \in \mathcal{A}_{n, 1}^{(1)}: \max _{1 \leq j \leq t} a_{j} \leq n-w\right\} .
\end{aligned}
$$

Thus, all $a \in \mathcal{A}_{n, 1}^{(2)}$ have at least one very large partial quotient $a_{j}$; on the other hand, all $a_{j}$ 's for $a \in \mathcal{A}_{n, 2}^{(2)}$ are relatively small.

Next we split $\mathcal{A}_{n, 1}^{(2)}$ into the set where the largest partial quotient is the last one and where it is not:

$$
\begin{aligned}
& \mathcal{A}_{n, 1}^{(3)}=\left\{a=\left(a_{1}, \ldots, a_{t}\right) \in \mathcal{A}_{n, 1}^{(2)}: a_{t}>\max \left\{a_{1}, \ldots, a_{t-1}\right\}\right\}, \\
& \mathcal{A}_{n, 2}^{(3)}=\mathcal{A}_{n, 1}^{(2)} \backslash \mathcal{A}_{n, 1}^{(3)}=\left\{a=\left(a_{1}, \ldots, a_{t}\right) \in \mathcal{A}_{n, 1}^{(2)}: a_{t} \leq \max \left\{a_{1}, \ldots, a_{t-1}\right\}\right\} .
\end{aligned}
$$

The split of $\mathcal{A}_{n}$ into subsets is shown in Figure 2.

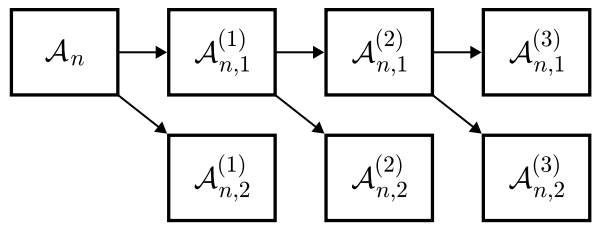

Fig. 2. Split of the index set $\mathcal{A}_{n}$

Additionally, we split the sum $\Sigma_{n, 1}^{(3)}$ as

$\Sigma_{n, 1}^{(3)}=\Sigma_{n, 1}^{(3)+}+\Sigma_{n, 1}^{(3)-} \quad$ with $\quad \Sigma_{n, 1}^{(3)+}=\sum_{a \in \mathcal{A}_{n, 1}^{(3)}} \frac{1}{\left(q q_{+}\right)^{\beta}}, \Sigma_{n, 1}^{(3)-}=\sum_{a \in \mathcal{A}_{n, 1}^{(3)}} \frac{1}{\left(q q_{-}\right)^{\beta}}$.

As a result, we have the following split of the sum $\sigma_{\beta}\left(\mathcal{F}_{n}\right)$ defined in (7):

$$
\sigma_{\beta}\left(\mathcal{F}_{n}\right)=\Sigma_{n, 2}^{(1)}+\Sigma_{n, 2}^{(2)}+\Sigma_{n, 2}^{(3)}+\Sigma_{n, 1}^{(3)+}+\Sigma_{n, 1}^{(3)-} .
$$

In Lemma 3 we consider the sum $\Sigma_{n, 2}^{(1)}$ (which accounts for all the terms with very large denominators) and establish that $\Sigma_{n, 2}^{(1)} \leq n^{-(2 \beta-1)}$ for all $n$. In Lemma 5 we prove that $\Sigma_{n, 2}^{(2)}+\Sigma_{n, 2}^{(3)} \ll(\log n)^{2 \beta} / w^{2 \beta-1}$ as $n \rightarrow \infty$. In Lemma 6 we demonstrate that $\Sigma_{n, 1}^{(3)+} \ll n^{-2 \beta}$ as $n \rightarrow \infty$; this sum is obviously asymptotically dominated by the cumulative effect of the others. 
Finally, in Lemma 7 we prove that

$$
\Sigma_{n, 1}^{(3)-}=\frac{1}{n^{\beta}} \cdot \frac{2 \zeta(2 \beta-1)}{\zeta(2 \beta)}+O\left(\frac{1}{n^{2 \beta-1}}+\frac{w}{n^{\beta+1}}+\frac{1}{n^{\beta} w^{2(\beta-1)}}\right) \quad \text { as } n \rightarrow \infty .
$$

Therefore, the decomposition (9) and Lemmas 3, 5, 6 and 7 imply the following asymptotic expansion for the sum $\sigma_{\beta}\left(\mathcal{F}_{n}\right)$ :

$$
\sigma_{\beta}\left(\mathcal{F}_{n}\right)=\frac{2 \zeta(2 \beta-1)}{n^{\beta} \zeta(2 \beta)}+O\left(\frac{1}{n^{2 \beta-1}}+\frac{(\log n)^{2 \beta}}{w^{2 \beta-1}}+\frac{w}{n^{\beta+1}}+\frac{1}{n^{\beta} w^{2(\beta-1)}}\right)
$$

The choice of $w=w(n, \beta)$ in accordance with (8) provides the minimum error term which is $O\left(n^{-(\beta+1)(2 \beta-1) /(2 \beta)} \log n\right)$, as stated in (2). This result is an easy consequence of the fact that the sum of the second and third terms in $O(\cdot)$ dominates the first and fourth terms. Note also that $(\beta+1)(2 \beta-1) /(2 \beta)>\beta$ for all $\beta>1$ so that the error term is smaller than the main term in this range of $\beta$.

\subsection{Lemmas}

Lemma 2. Let $n \geq 2, a=\left(a_{1}, \ldots, a_{t}\right) \in \mathcal{A}_{n}, q=q(a)=\left[a_{1}, \ldots, a_{t}\right]$, and $q_{-}, q_{+}$be as defined in (5). We have:

$$
\begin{gathered}
q=q_{-}+q_{+} \leq n q_{-}, \\
q_{-} \leq q_{+} \leq a_{t} q_{-}, \\
\sum_{a \in \mathcal{A}_{n}}\left(\frac{1}{q q_{-}}+\frac{1}{q q_{+}}\right)=1, \\
\frac{1}{\left(q q_{-}\right)^{\beta}}+\frac{1}{\left(q q_{+}\right)^{\beta}} \leq \frac{n^{\beta-1}}{q^{2(\beta-1)}}\left(\frac{1}{q q_{-}}+\frac{1}{q q_{+}}\right) .
\end{gathered}
$$

Proof. The equality in (10) follows from the definition of $\mathcal{Q}_{n}$. For $n=2$ the inequalities in (10) and (11) can be easily checked directly. For $n>2$ these inequalities follow from (5) and the standard recurrence for the continuants of successive continued fraction convergents. The formula (12) expresses the fact that the total length of all the intervals in the partition generated by the points in $\mathcal{F}_{n}$ is 1 .

Let us now prove (13). Using the inequalities $q_{+} \geq q_{-}$and $q_{-} \geq q / n$ (see (10) and (11)), for all $a \in \mathcal{A}_{n}$ we obtain

$$
\begin{aligned}
\frac{1}{\left(q q_{-}\right)^{\beta}}+\frac{1}{\left(q q_{+}\right)^{\beta}} & \leq\left(\frac{1}{q q_{-}}+\frac{1}{q q_{+}}\right) \max \left\{\frac{1}{\left(q q_{-}\right)^{\beta-1}}, \frac{1}{\left(q q_{+}\right)^{\beta-1}}\right\} \\
& =\frac{1}{\left(q q_{-}\right)^{\beta-1}}\left(\frac{1}{q q_{-}}+\frac{1}{q q_{+}}\right) \leq \frac{n^{\beta-1}}{q^{2(\beta-1)}}\left(\frac{1}{q q_{-}}+\frac{1}{q q_{+}}\right) .
\end{aligned}
$$

Lemma 3. For all $n \geq 1$ we have $\Sigma_{n, 2}^{(1)} \leq n^{-(2 \beta-1)}$. 
Proof. As $q \geq n^{r}$ for $a \in \mathcal{A}_{n, 2}^{(1)}$, using the equality (12), the inequality (13) and the definition of $r$ we obtain

$$
\begin{aligned}
\Sigma_{n, 2}^{(1)} & \leq \frac{n^{\beta-1}}{q^{2(\beta-1)}} \sum_{a \in \mathcal{A}_{n, 2}^{(1)}}\left(\frac{1}{q q_{-}}+\frac{1}{q q_{+}}\right) \leq \frac{n^{\beta-1}}{q^{2(\beta-1)}} \leq \frac{n^{\beta-1}}{n^{2 r(\beta-1)}} \\
& =\frac{1}{n^{(\beta-1)(2 r-1)}}=\frac{1}{n^{2 \beta-1}} .
\end{aligned}
$$

LEMmA 4. For all $a=\left(a_{1}, \ldots, a_{t}\right) \in \mathcal{A}_{n, 1}^{(1)}$ with $n \geq 2$ we have $t \leq$ $C \log n$, where $C=C(\beta)=r \log ((\sqrt{5}+1) / 2)$.

Proof. This follows from the fact that for all $a=\left(a_{1}, \ldots, a_{t}\right) \in \mathcal{A}_{n, 1}^{(1)}$ we have

$$
\left(\frac{\sqrt{5}+1}{2}\right)^{t} \leq\left[a_{1}, \ldots, a_{t}\right] \leq n^{r}
$$

Lemma 5. As $n \rightarrow \infty$, we have

$$
\Sigma_{n, 2}^{(2)}+\Sigma_{n, 2}^{(3)} \ll \frac{(\log n)^{2 \beta}}{w^{2 \beta-1}} .
$$

Proof. Lemma 4 states that for all $a \in \mathcal{A}_{n, 1}^{(1)}$ (that is, when $q(a) \leq n^{r}$ ) we have $t \leq C \log n$. As $n=a_{1}+\cdots+a_{t} \leq t \max a_{j}$, this implies $\max a_{j} \geq$ $n / C \log n$.

Let $a=\left(a_{1}, \ldots, a_{t}\right) \in \mathcal{A}_{n, 2}^{(2)}$ and $j$ be such that $a_{j}=\max \left\{a_{1}, \ldots, a_{t}\right\}$. Since $a_{j} \leq n-w$, for the sum of the remaining $a_{i}$ 's we have $\sum_{i \neq j} a_{i}>w$ and similarly to the above, the second largest value of $a_{i}$ 's is larger than or equal to $w / C \log n$. This implies that for any $a=\left(a_{1}, \ldots, a_{t}\right) \in \mathcal{A}_{n, 2}^{(2)}$, there exist two indices $1 \leq k \neq l \leq t$ such that $a_{k} \geq w / C \log n$ and $a_{l} \geq w / C \log n$ and therefore for at least one index $j \leq t-1$ we have $a_{j} \geq w / C \log n$.

If $a=\left(a_{1}, \ldots, a_{t}\right) \in \mathcal{A}_{n, 2}^{(3)}$ there is $j<t$ such that $a_{j}=\max \left\{a_{1}, \ldots, a_{t}\right\}$. Since $\mathcal{A}_{n, 2}^{(3)} \subseteq \mathcal{A}_{n, 1}^{(2)}$, for this $a_{j}$ we have $a_{j}>n-w>w / \log n$ for all $n \geq 3$.

Set $c=\max \{1,1 / C\}$ and let $n \geq 3$. Then for all $a=\left(a_{1}, \ldots, a_{t}\right) \in$ $\mathcal{A}_{n, 2}^{(2)} \cup \mathcal{A}_{n, 2}^{(3)}$ we have $a_{j} \geq c w / \log n$ for at least one $j \leq t-1$.

Therefore,

$$
\Sigma_{n, 2}^{(2)}+\Sigma_{n, 2}^{(3)} \leq 2 \sum_{\substack{a_{1}+\cdots+a_{t-1} \leq n \\ \exists j: a_{j} \geq c w / \log n}}\left(\frac{1}{\left(q q_{-}\right)^{\beta}}+\frac{1}{\left(q q_{+}\right)^{\beta}}\right) \leq 4 \sum_{\substack{a_{1}+\cdots+a_{t-1} \leq n \\ \exists j: a_{j} \geq c w / \log n}} \frac{1}{\left(q q_{-}\right)^{\beta}} .
$$

(Here we have also used the fact that $q_{+} \geq q_{-}$.) Clearly,

$$
\begin{gathered}
q>a_{j} a_{t} \cdot\left[a_{1}, \ldots, a_{j-1}\right] \cdot\left[a_{j+1}, \ldots, a_{t-1}\right], \\
q_{+} \geq q_{-}>a_{j} \cdot\left[a_{1}, \ldots, a_{j-1}\right] \cdot\left[a_{j+1}, \ldots, a_{t-1}\right] .
\end{gathered}
$$




\section{Hence}

$$
\begin{aligned}
& \Sigma_{n, 2}^{(2)}+\Sigma_{n, 2}^{(3)} \\
& \leq \sum_{j<C \log n} \sum_{c w / \log n<a_{j} \leq n} \sum_{\substack{a=\left(a_{1}, \ldots, a_{t}\right) \in \mathcal{A}_{n, 1}^{(1)} \\
a_{j} \text { fixed } ; \leq t-1}} \frac{1}{a_{j}^{2 \beta}\left[a_{1}, \ldots, a_{j-1}\right]^{2 \beta}\left[a_{j+1}, \ldots, a_{t-1}\right]^{2 \beta} a_{t}^{\beta}} \\
& \leq \frac{4 C \log n}{(c w / \log n)^{2 \beta-1}} \sum_{u+v \leq w} \sum_{a_{1}+\cdots+a_{r}=u} \frac{1}{\left[a_{1}, \ldots, a_{r}\right]^{2 \beta}} \sum_{b_{1}+\cdots+b_{h}=v} \frac{1}{\left[b_{1}, \ldots, b_{h-1}\right]^{2 \beta} b_{h}^{\beta}} \\
& \leq \frac{4 C(\log n)^{2 \beta}}{(c w)^{2 \beta-1}} \sum_{a_{1}+\cdots+a_{r} \leq w} \frac{1}{\left[a_{1}, \ldots, a_{r}\right]^{2 \beta}} \sum_{b_{1}+\cdots+b_{h-1} \leq w} \frac{1}{\left[b_{1}, \ldots, b_{h-1}\right]^{2 \beta}} \sum_{b_{h}=1}^{\infty} \frac{1}{b_{h}^{\beta}} \\
& =\frac{4 C \zeta(\beta)}{c^{2 \beta-1}} \cdot \frac{(\log n)^{2 \beta}}{w^{2 \beta-1}}\left(\sum_{a_{1}+\cdots+a_{r} \leq w} \frac{1}{\left[a_{1}, \ldots, a_{r}\right]^{2 \beta}}\right)^{2} .
\end{aligned}
$$

Since

$$
\sum_{a_{1}+\cdots+a_{r} \leq w} \frac{1}{\left[a_{1}, \ldots, a_{r}\right]^{2 \beta}} \leq \sum_{q=1}^{\infty} \frac{\varphi(q)}{q^{2 \beta}}=\frac{\zeta(2 \beta-1)}{\zeta(2 \beta)}
$$

we obtain

$$
\Sigma_{n, 2}^{(2)}+\Sigma_{n, 2}^{(3)} \leq \frac{4 C \zeta(\beta)}{c^{2 \beta-1}}\left(\frac{\zeta(2 \beta-1)}{\zeta(2 \beta)}\right)^{2} \frac{(\log n)^{2 \beta}}{w^{2 \beta-1}},
$$

and the lemma follows.

Lemma 6. As $n \rightarrow \infty$, we have $\Sigma_{n, 1}^{(3)+} \ll n^{-2 \beta}$.

Proof. Since $q_{+}<q \leq n q_{-}=n\left[a_{1}, \ldots, a_{t-1}\right]$ for all $a=\left(a_{1}, \ldots, a_{t}\right) \in \mathcal{A}$, we have

$$
\begin{aligned}
\Sigma_{n, 1}^{(3)+} & =\sum_{\substack{a_{1}+\cdots+a_{t}=n \\
a_{t}>n-w, q<n^{r}}} \frac{1}{\left(q q_{+}\right)^{\beta}} \leq \sum_{n-w<a_{t} \leq n} \sum_{a_{1}+\cdots+a_{t-1}=n-a_{t}} \frac{1}{\left(q q_{+}\right)^{\beta}} \\
& \leq \frac{1}{(n-w)^{2 \beta}} \sum_{a_{1}+\cdots+a_{t-1} \leq w} \frac{1}{\left[a_{1}, \ldots, a_{t-1}\right]^{2 \beta}} \\
& \leq \frac{2}{(n-w)^{2 \beta}} \sum_{q=1}^{\infty} \frac{\varphi(q)}{q^{2 \beta}} \ll n^{-2 \beta} .
\end{aligned}
$$

Lemma 7. As $n \rightarrow \infty$, we have

$$
\Sigma_{n, 1}^{(3)-}=\frac{1}{n^{\beta}} \cdot \frac{2 \zeta(2 \beta-1)}{\zeta(2 \beta)}+O\left(\frac{1}{n^{2 \beta-1}}+\frac{w}{n^{\beta+1}}+\frac{1}{n^{\beta} w^{2(\beta-1)}}\right) .
$$


Proof. We have

$$
\begin{aligned}
\Sigma_{n, 1}^{(3)-} & =\sum_{\substack{a=\left(a_{1}, \ldots, a_{t}\right) \in \mathcal{A}_{n} \\
q(a)<n^{r}, a_{t}>n-w}} \frac{1}{\left(q(a) q_{-}(a)\right)^{\beta}} \\
& =\sum_{\substack{a=\left(a_{1}, \ldots, a_{t}\right) \in \mathcal{A}_{n} \\
a_{t}>n-w}} \frac{1}{\left(q(a) q_{-}(a)\right)^{\beta}}-\sum_{\substack{a=\left(a_{1}, \ldots, a_{t}\right) \in \mathcal{A}_{n} \\
q(a) \geq n^{r}, a_{t}>n-w}} \frac{1}{\left(q(a) q_{-}(a)\right)^{\beta}} .
\end{aligned}
$$

The second sum is

$$
\sum_{\substack{a_{1}+\cdots+a_{t}=n \\ q(a) \geq n^{r}, a_{t}>n-w}} \frac{1}{\left(q(a) q_{-}(a)\right)^{\beta}}=O\left(n^{-(2 \beta-1)}\right) \quad \text { as } n \rightarrow \infty
$$

and for the first sum we have

$$
\sum_{\substack{a=\left(a_{1}, \ldots, a_{t}\right) \in \mathcal{A}_{n} \\
a_{t}>n-w}} \frac{1}{\left(q(a) q_{-}(a)\right)^{\beta}}=\sum_{\begin{array}{c}
a=\left(a_{1}, \ldots, a_{t}\right) \in \mathcal{A}_{n} \\
a_{1}+\cdots+a_{t-1} \leq w
\end{array}} \frac{1}{(q(a) q-(a))^{\beta}} .
$$

In the Farey tree $\mathcal{F}_{n}$, each Farey fraction with denominator $q_{-}(a)=$ $\left[a_{1}, \ldots, a_{t-1}\right]$ such that $a_{1}+\cdots+a_{t-1}<n$ is a neighbour to two Farey fractions with denominators $q(a)=\left[a_{1}, \ldots, a_{t}\right]$ with $a_{1}+\cdots+a_{t}=n$ (see $\left.(6)\right)$. Additionally, since

$$
q=a_{t} q_{-}+\left(q_{-}\right)_{-}=q_{-}(n+O(w)) \quad \text { as } n \rightarrow \infty,
$$

we have

$$
\frac{1}{\left(q(a) q_{-}(a)\right)^{\beta}}=\frac{1}{n^{\beta} q_{-}^{2 \beta}}\left(1+O\left(\frac{w}{n}\right)\right) .
$$

In view of these two facts we obtain

$$
\begin{aligned}
& \Sigma_{n, 1}^{(3)-}=\frac{2}{n^{\beta}} \sum_{\substack{a_{1}+\cdots+a_{t-1} \leq w \\
a_{t-1}>1 \text { for } t>2}} \frac{1}{\left[a_{1}, \ldots, a_{t-1}\right]^{2 \beta}}+O\left(n^{-(2 \beta-1)}+\frac{w}{n^{\beta+1}}\right) \\
& =\frac{2}{n^{\beta}} \sum_{q=1}^{\infty} \frac{\varphi(q)}{q^{2 \beta}}+O\left(\frac{1}{n^{2 \beta-1}}+\frac{w}{n^{\beta+1}}+n^{-\beta} \sum_{a_{1}+\cdots+a_{t-1} \geq w} \frac{1}{\left[a_{1}, \ldots, a_{t-1}\right]^{2 \beta}}\right) .
\end{aligned}
$$

As

$$
\sum_{q=1}^{\infty} \frac{\varphi(q)}{q^{2 \beta}}=\frac{\zeta(2 \beta-1)}{\zeta(2 \beta)}
$$

and

$$
\sum_{a_{1}+\cdots+a_{i} \geq w} \frac{1}{\left[a_{1}, \ldots, a_{i}\right]^{2 \beta}} \ll \sum_{q \geq w} \frac{1}{q^{2 \beta-1}} \ll w^{-2(\beta-1)},
$$

the lemma follows. 
Acknowledgments. The authors are grateful to our collegues M. N. Huxley (Cardiff), P. Kargaev (St. Petersburg), L. Pronzato (Antibes) and H. P. Wynn (London) for useful discussions. The authors are also grateful to the referee for valuable comments.

\section{References}

V. Drobot (1981), Uniform partitions of an interval, Trans. Amer. Math. Soc. 268, 151-160.

R. R. Hall (1970), A note on Farey series, J. London Math. Soc. (2), 2, 139-148.

S. Kanemitsu, R. Sitaramachandra Rao and A. Siva Rama Sarma (1982), Some sums involving Farey fractions, I, J. Math. Soc. Japan 34, 125-142.

J. C. Lagarias (1992), Number theory and dynamical systems, Proc. Sympos. Appl. Math. $46,35-72$.

T. Prellberg and J. Slawny (1992), Maps of intervals with indifferent fixed points: thermodynamic formalism and phase transitions, J. Statist. Phys. 66, 503-514.

L. Pronzato, H. P. Wynn and A. A. Zhigljavsky (1999), Dynamical Search, CRC/Chapman and Hall.

M. Schroeder (1991), Fractals, Chaos, Power Laws, Freeman, New York.

B. Vallée (2001), Dynamical sources in information theory: fundamental intervals and word prefixes, Algorithmica 29, 262-306.

Faculty of Mechanics and Mathematics

Moscow State University

119899 Moscow, Russia

E-mail: moshche@mech.math.msu.su
Mathematics Institute Cardiff University

Cardiff CF24 4YH, UK

E-mail: ZhigljavskyAA@cardiff.ac.uk

Received on 14.2.2003

and in revised form on 4.9.2003 